\title{
Synthesis of most polyene natural product motifs using just twelve building blocks and one coupling reaction
}

\author{
Eric M. Woerly, Jahnabi Roy, and Martin D. Burke \\ Howard Hughes Medical Institute, University of Illinois at Urbana-Champaign, Urbana, IL 61801, \\ USA
}

\section{Abstract}

The inherent modularity of polypeptides, oligonucleotides, and oligosaccharides has been harnessed to achieve generalized building block-based synthesis platforms. Importantly, like these other targets, most small molecule natural products are biosynthesized via iterative coupling of bifunctional building blocks. This suggests that many small molecules also possess inherent modularity commensurate with systematic building block-based construction. Supporting this hypothesis, here we report that the polyene motifs found in $>75 \%$ of all known polyene natural products can be synthesized using just 12 building blocks and one coupling reaction. Using the same general retrosynthetic algorithm and reaction conditions, this platform enabled the synthesis of a wide range of polyene frameworks covering all of this natural product chemical space, and first total syntheses of the polyene natural products asnipyrone $\mathrm{B}$, physarigin $\mathrm{A}$, and neurosporaxanthin $\beta$-D-glucopyranoside. Collectively, these results suggest the potential for a more generalized approach for making small molecules in the laboratory.

\section{Introduction}

Most of the molecules found in living systems are naturally prepared via the iterative assembly of a limited number of bifunctional building blocks (Figure 1a). ${ }^{1}$ For example, proteins are usually derived from just 20 amino acids, and DNA and RNA are each made from only 4 nucleotides. ${ }^{1}$ Capitalizing on this inherent modularity, generalized building block-based strategies have been developed for efficient, flexible, and fully-automated access to these molecules in the laboratory, ${ }^{2-4}$ and the resulting impacts on science, medicine, and technology have been transformational. In a similar vein, a recent analysis revealed that $75 \%$ of all mammalian oligosaccharides are comprised of just 36 monosaccharide units, ${ }^{5}$ and an increasingly general platform for automated oligosaccharide synthesis is broadening access to this class of biomolecules as well. ${ }^{6}$ In stark contrast, the laboratory synthesis of small molecule natural products has traditionally involved the customized development of a unique pathway and collection of building blocks to access

\footnotetext{
Users may view, print, copy, and download text and data-mine the content in such documents, for the purposes of academic research, subject always to the full Conditions of use:http://www.nature.com/authors/editorial_policies/license.html\#terms

Author Contributions E.M.W. and M.D.B. conceived the project. E.M.W., J.R., and M.D.B. designed and executed experiments. E.M.W. and M.D.B. wrote the paper.

Additional Information The University of Illinois has filed patents on MIDA boronate chemistry. Full experimental details, procedures and characterization for new compounds are included in the Supplementary Information.
} 
each target. As a result, despite many important advances in accessing individual structures, small molecule synthesis remains a relatively inefficient and inflexible process that is practiced almost exclusively by specialists.

Importantly, like their peptide, oligonucleotide, and oligosaccharide counterparts, most small molecule natural products are biosynthesized via iterative building block assembly (Figure 1b). ${ }^{1}$ For example, polyketides are constructed from malonyl- and methylmalonylCoA, polyterpenes from isopentenyl and dimethylallyl pyrophosphate, fatty acids from malonyl CoA, non-ribosomal peptides from amino acids, and polyphenylpropanoids from phenylpropane subunits. This suggests that a systematic building block-based approach for making many small molecules should likewise be attainable.

With this goal in mind, we aim to identify substructural motifs that are prevalent in natural products and transform them into minimized collections of bifunctional building blocks that are compatible with iterative assembly. ${ }^{7-10}$ Polyene natural products provide an excellent opportunity for exploring this concept, as they are well represented across all major biosynthetic classes (Figure 1c). ${ }^{11}$ These molecules also perform a wide range of important functions including serving as pharmaceutical agents, ${ }^{12}$ pigments for light harvesting, ${ }^{13}$ fluorescent probes, ${ }^{14}$ quenchers of reactive oxygen species, ${ }^{15}$ and transducers of solar energy into mechanical energy. ${ }^{16}$ Synthesis of these natural products is made challenging by their sensitivities to light, oxygen, and many common reagents including protic and Lewis acids, as well as difficulties in controlling the stereochemistry of each double bond.

Common methods for the synthesis of polyenes include olefin-generating reactions, ${ }^{11}$ e.g., the Wittig, Horner-Wadsworth-Emmons, and Julia olefinations, but these stereoselective processes often suffer from a lack of stereocontrol. Transition metal-based reactions ${ }^{17,18}$ with organozinc, organostannane, organosilicon, and organoboron intermediates have the important advantage of stereospecificity, i.e., the stereochemistry in the building blocks can be faithfully translated into the products, but the required building blocks and/or intermediates often suffer from instability and/or toxicity.

The preparation of polyenes via cross-coupling of non-toxic organoboron intermediates have specifically been impeded by the instability of polyenylboronic acids, ${ }^{19}$ yet some progress has been made, including several recent examples involving iterative cross-coupling with stable $N$-methyliminodiacetic acid (MIDA) boronates. ${ }^{20-23}$ In this type of synthesis, bifunctional haloboronic acid building blocks having all of the required functional groups pre-installed in the correct oxidation states and with the desired stereochemical relationships are sequentially linked together using only the stereospecific Suzuki-Miyaura cross-coupling reaction in an iterative manner. The MIDA ligand prevents random oligomerization via reversible attenuation of the reactivity of the borane terminus, ${ }^{21,22}$ analogous to the way an FMOC group enables the precise assembly of amino acids. MIDA boronates are also convenient to prepare, analyze, purify, and store and more than 170 are now commercially available. ${ }^{24}$ Collectively, these features have enabled the simple, efficient, and flexible synthesis of a wide range of small molecule natural products, ${ }^{21,23,25-30}$ pharmaceutical agents, ${ }^{31-34}$ ligands, ${ }^{35,36}$ and materials ${ }^{37}$ via iterative cross-coupling. 
Building on this momentum, we decided to ask the question: how many bifunctional MIDA boronate building blocks would be required to make most of the polyene motifs found in nature? To find the answer, we devised a general retrosynthetic algorithm for systematically deconstructing these motifs into the minimum total number of building blocks. This analysis generated the intriguing hypothesis that the polyene motifs found in $>75 \%$ of all polyene natural products can be prepared using just 12 MIDA boronate building blocks and one coupling reaction. As described below, we have tested and confirmed this hypothesis.

\section{Results}

The Dictionary of Natural Products, ${ }^{38}$ a comprehensive database of small molecules isolated from natural sources and characterized to date (238,541 as of 1/15/2012), was used to identify all known polyene natural products. Specifically, a substructure search for a polyene, defined as three or more carbon-carbon double bonds in conjugation, none of which are contained in a <12-membered ring, returned 2,839 natural products. Importantly, this set includes small molecules derived from all major biosynthetic classes: polyterpenes, polyketides, fatty acids, hybrid peptide/polyketides, and polyphenylpropanoids (Figure 1c).

To determine the minimum number of bifunctional MIDA boronate building blocks that would be required to access the polyene motifs found in most of these natural products and maximize the stability of the corresponding building blocks and intermediates, we developed the following systematic 3-step retrosynthetic algorithm: 1) The polyene motif is identified as the polyene framework minus the two olefinic termini. 2) Using mono-, di-, and triene haloalkenyl MIDA boronates, the polyene motif is dissected into the fewest number of building blocks that are as similar in size as possible. 3) Disconnections are chosen such that the length of the longest polyenyl borane intermediate in each pathway is minimized.

The following examples demonstrate the application of this general retrosynthetic algorithm to three structurally diverse and previously not synthesized polyene natural products derived from a range of biosynthetic pathways (Figure 2). Asnipyrone B is a polyketide derived polyene natural product isolated from Aspergillus niger ${ }^{39}$ (Figure 2a). Applying the algorithm, the targeted motif is identified as a simple transmonoene, and a single bifunctional building block would be required. Importantly, this same substructure is also found in more than 1,200 other polyene natural products, including epolactaene, apozeaxanthione, and dihydroxyagerafastin (Figure 1c), revealing that the same bifunctional building block could contribute to the construction of many other natural products.

Using the same algorithm, the polyene motif of the fatty acid derived natural product physarigin $\mathrm{A}^{40}$ is identified as a tetraene, which is then dissected into two copies of the same dienyl building block (Figure 2b). This dienyl substructure is also found in more than 350 additional polyene natural products including auxarconjugatin A, natamycin, and fuligoic acid (Figure 1c), again revealing the potential for a high degree of overlapping building block utility.

In a third example, the complex polyene motif found in the polyterpene derived natural product neurosporaxanthin $\beta$-D-glucopyranoside ${ }^{41}$ is identified as the corresponding octaene 
(Figure 2c). Following the general retrosynthetic guidelines, this motif is dissected into the fewest number of building blocks of similar size, i.e., a diene and two trienes. In order to minimize the length and therefore maximize the stability of the polyenyl borane intermediates, the algorithm selects first coupling a diene building block followed by two different trienes. As a result, three building blocks are identified as being required for this synthesis. Importantly, at least one of these building blocks is similarly identified as potentially contributory to the synthesis of over 1,100 other polyene natural products including violaxanthin, enacyloxin IIa, and hemicalyculin A (Figure 1c), again demonstrating the potential for a high degree of redundant building block utilization.

Systematic manual application of this same algorithm to all 2,839 polyene natural products and filtering for maximum overlap in building block utilization revealed a striking result: only 12 bifunctional haloalkenyl MIDA boronate building blocks (BB1-BB12, Figure 3a) are required to theoretically access the polyene motifs found in $>75 \%$ of polyene natural products. Efficient and stereospecific syntheses of all 12 of these building blocks was readily accomplished by taking advantage of many of the enabling features of the MIDA boronate platform, including compatibility of the MIDA boronate group to many different types of reaction conditions and purification by column chromatography (see Supporting Information), and four of these building blocks (BB1-BB4) are already commercially available.

When we first attempted to construct complex polyene motifs via iteratively coupling these building blocks together, we encountered an important limitation. ${ }^{21}$ Specifically, while the polyenyl MIDA boronate intermediates were stable, many of the deprotected polyenyl boronic acid intermediates were not, ${ }^{25,26}$ resulting in poor or no yields of the desired final products. Alternatively, we found that the corresponding polyenylboronic esters, which can also be readily formed via transligation of MIDA boronates with pinacol, are much more stable. The challenge with using pinacol esters as intermediates, however, is that they generally require aqueous basic conditions for subsequent cross-coupling, ${ }^{42}$ and such conditions are incompatible with the aqueous base-labile MIDA boronate protecting group on the bifunctional building blocks. Suggesting an opportunity to overcome this potential impasse, it was determined that when DMSO is employed as solvent, vinyl pinacol boronic esters can be cross-coupled under anhydrous conditions. ${ }^{26,43}$ We thus envisioned a modified iterative cross-coupling cycle involving the initial coupling of a pinacol boronic ester to a bifunctional halo MIDA boronate building block under anhydrous DMSO conditions followed by a deprotection of the resulting MIDA boronate to generate a new pinacol boronic ester suitable for the next round of coupling (Figure $3 b$ ).

With the goal of developing a maximally general platform, we further sought to identify common reaction conditions for coupling polyenyl pinacol esters with haloalkenyl MIDA boronate building blocks and for transforming the resulting polyenyl MIDA boronates into the corresponding pinacol boronic esters. For the latter, we found that a wide range of polyenyl MIDA boronates of varying lengths and with varying substitution patterns can all be converted to the corresponding pinacol esters using the same conditions: pinacol and $\mathrm{NaHCO}_{3}$ in $\mathrm{MeOH}$ at $45^{\circ} \mathrm{C}$ for $3 \mathrm{~h}$. Moreover, we found that polyenyl pinacol esters of varying length, stereochemistry, and methyl substitution patterns can all be coupled to 
mono-, di- and trienyl bromide and iodide bifunctional MIDA boronate building blocks using the same set of cross-coupling conditions: XPhos palladacycle pre-catalyst, ${ }^{44} \mathrm{Cs}_{2} \mathrm{CO}_{3}$, and DMSO. It was further determined that final couplings between a polyenyl MIDA boronate intermediate and capping vinyl halide building block can be best achieved using a common set of aqueous basic conditions (XPhos palladacycle pre-catalyst, $\mathrm{NaOH}$, and THF: $\mathrm{H}_{2} \mathrm{O}$ ) to promote the in situ release of an unstable but also highly reactive polyenyl boronic acid directly from the corresponding stable polyenyl MIDA boronate. ${ }^{45}$

With these building blocks and general reaction conditions in hand, we sought to test the hypothesis that they can collectively enable preparation of most of the polyenes found in nature. Enabling such an experiment, we identified a collection of polyene motifs that represent the corresponding chemical space occupied by $>75 \%$ of all polyene natural products (A-O, Figure 4). This collection includes both trans- and cis-olefins, various methyl substitution patterns, and a wide range of chain lengths (from three to ten double bonds), and thus represents a substantial challenge for the development of a common synthesis platform.

Utilizing only the aforementioned general retrosynthetic algorithm, the same twelve MIDA boronate building blocks, and common deprotection and cross-coupling conditions, we attempted to synthesize all of the targeted polyene motifs 1-15 with MIDA boronate 16 and vinyl iodide $\mathbf{1 7}$ serving as representative natural product-like capping groups. Without any ad hoc optimization of the platform, all of the targeted polyene motifs were successfully prepared (Figure 5).

These syntheses ranged from a single round of iterative cross-coupling to generate triene 1 to four iterations to generate highly complex decaene $\mathbf{1 5}$. The yields for the deprotection steps were generally outstanding, even with very long and complex polyene intermediates. Importantly, while the yields for the cross-couplings tended to decrease somewhat as the length of the polyene intermediates grew larger, the predictability of this trend enabled us to begin each sequence with the appropriate amounts of the required building blocks and thereby produce milligram quantities of the targeted final products in every case. Successful syntheses of these targets demonstrates a general approach to the polyene motifs found in $>75 \%$ of all the polyene natural products isolated to date.

Encouraged by the broad applicability of this platform, we further targeted its application to complete the first total syntheses of a series of polyene natural products representing a range of biosynthetic pathways, i.e., the polyketide asnipyrone B, the fatty acid physarigin A, and the polyterpene neurosporaxanthin $\beta$-D-glucopyranoside, using the same retrosynthetic algorithm, collection of bifunctional building blocks, and common reaction conditions (NP1-NP3, Figure 6). Applying this approach to each of these total syntheses therefore only required preparation of the corresponding capping elements. Thus, relative to the traditional design of an individualized synthesis route to each natural product, preparation of all the corresponding customized building blocks, and ad hoc optimization of all the reaction conditions required for coupling those building blocks together, this systematized approach has major advantages. Moreover, as described below, more than $75 \%$ of the capping elements represented in the complete collection of polyene natural products fall into just 20 
general structural categories for which common synthetic routes exist or can be readily envisioned.

After facile preparation of the required capping elements (Supporting Information), ${ }^{46,47}$ preparation of asnipyrone B NP1 commenced with a standardized deprotection of MIDA boronate 18 and coupling with commercially available halo MIDA boronate $\mathbf{B B 3}$ to provide diene 19 (Figure 6a). It is notable that this sterically encumbered 1,3-methyl-substituted motif was readily prepared without modification of the general reaction conditions. Application of the general in situ deprotection/coupling conditions with capping building block 20 completed a very efficient and fully stereocontrolled first total synthesis of this natural product.

Physarigin A NP2 similarly only required preparation of the capping building blocks 21 and 22. The latter is a variant of a known building block, ${ }^{48}$ and preparation of the former was greatly facilitated by using another commercially-available MIDA boronate (Supporting Information) (Figure 6b). ${ }^{49}$ In the event, deprotection of $\mathbf{2 1}$ and cross-coupling to $\mathbf{B B 5}$ using our standardized conditions provided the triene $\mathbf{2 3}$ in good yield. Another round of deprotection and coupling with the same bifunctional building block established the structure of the targeted pentaene intermediate $\mathbf{2 4}$. A final cross-coupling with halide $\mathbf{2 2}$ completed the first total synthesis of physarigin A.

Finally, we questioned whether this platform could enable the total synthesis of the complex polyene natural product neurosporaxanthin $\beta$-D-glucopyranoside NP3 (Figure 6c). The corresponding capping elements were again readily accessed (Supporting Information), and the synthesis commenced via deprotection of MIDA boronate 25 and subsequent crosscoupling of the resulting pinacol boronic ester with diene $\mathbf{B B 6}$ to provide tetraene 26. A second, third, and fourth iteration of couplings with building blocks BB11, BB9, and 27 respectively, without any ad hoc optimization of our previously established standard conditions, followed by removal of the protecting groups, readily provided the first synthetic access to this highly complex polyene natural product.

All of these results collectively support the conclusion that most polyene natural product motifs can now be prepared using just twelve building blocks and one coupling reaction.

\section{Discussion}

Herein we explicitly demonstrate the synthesis of the polyene motifs found in $>75 \%$ of all known polyene natural products. These natural products represent much of the diversity found in the complete collection. It is further encouraging to note that accessing $>90 \%$ of this chemical space would require only 25 building blocks, $>95 \%$ would require a total of 50 , and the motifs found in all polyene natural products could be accessed with only 75 building blocks (Supporting Information).

Completing total syntheses via this approach also requires access to the terminal capping elements. Importantly, similar to the high level of structural redundancy found in the polyene motifs, a systematic analysis of the capping elements has also revealed a substantial level of structural overlap (for complete analysis, see Supporting Information). For example, 
if a unique pair of building blocks were found in each of the 2,839 targets, 5,678 capping elements would be necessary. However, the high level of structural redundancy reduces this number to 1,942 unique building blocks, and just 604 of these building blocks would be needed to access $75 \%$ of the polyene natural products. Moreover, these 604 building blocks can be subdivided into just 20 common structural classes (for example, $\alpha / \beta$-unsaturated esters, allylic alcohols, and styrenes), where most members of each class can likely be accessed using small variations of a common synthetic route. Syntheses of many of these building blocks can further be facilitated using already commercially available MIDA boronates, including BB1-BB4, just as the capping element 18 in the synthesis of asnipyrone B was prepared in a single step from BB3. Finally, alkenyl MIDA boronates can be converted directly into alkenyl halides, ${ }^{27}$ allowing for rapid preparation of many halide capping elements from the corresponding readily accessible MIDA boronates.

The 2,839 natural products included in this study represent more than $1 \%$ of all the natural products isolated to date. It is stimulating to consider how many building blocks would be required to access most of the remaining $99 \%$. While the answer to this is not yet known, the strategy demonstrated herein, i.e., systematically identifying common motifs and transforming them into bifunctional building blocks compatible with iterative coupling, provides a roadmap for pursuing this problem. For example, we have identified 12 additional common structural motifs that are collectively present in more than 100,000 natural products (Supporting Information). Half of these motifs have already been transformed into bifunctional halo MIDA boronates that are now commercially available. Achieving the same goal with some of the others will require solutions to frontier methodological problems, including new chemistry for making and stereospecifically crosscoupling Csp3 boronates. In this vein, it is highly encouraging to note that there have been many recent advances in methods to prepare and stereoretentively cross-couple Csp3 boronic acids and/or their derivatives. ${ }^{50}$

With at least the conceptual framework for a more generalized approach to small molecule synthesis in hand, the prospective impact of fully automating the building block assembly process is increasingly clear. The achievement of such automation with peptides, ${ }^{2}$ oligonucleotides, ${ }^{3}$ and increasingly oligosaccharides ${ }^{4}$ has had a transformative impact on the respective areas of molecular science. The key to automating iterative synthesis is finding a way to automatically purify the intermediates, and in all of these previous cases, this goal has been achieved via solid-phase synthesis. ${ }^{2-4}$ However, unlike their peptide, oligonucleotide, and oligosaccharide counterparts, small molecules do not inherently possess a common functional group handle that can be used for attachment to a solid support. Thus, generally automating small molecule synthesis represents an exceptional problem that will require a distinct solution.

Finally, while we have herein focused on a generalized approach for making known polyene natural products, we note that combinatorial assembly of the building blocks derived from this analysis could provide access to many new polyene natural product-like compounds. Expanding this same concept to include building blocks algorithmically derived from all known natural products could provide practical access to large collections of natural product-like compounds for functional screening. 
In summary, the development of a more general and systematic building block-based approach for small molecule synthesis would substantially expedite and broaden access to the extraordinary functional potential that these molecules possess. The results presented herein demonstrate an actionable roadmap for pursuing this objective.

\section{Methods}

Full experimental details, procedures, and characterization for new compounds are included in the Supplementary Information.

\section{Supplementary Material}

Refer to Web version on PubMed Central for supplementary material.

\section{Acknowledgements}

We gratefully acknowledge Adam Hill for helping to complete the synthesis of physarigin A and Sean O'Hara and Seiko Fujii for building block synthesis, as well as the NIH (GM080436 and GM090153) and HHMI for funding. M.D.B. is an HHMI Early Career Scientist and E.M.W. was an NSF Predoctoral Fellow.

\section{References}

1. Garret, RH.; Grisham, CM. Biochemistry. Saunders College Publishing; Fort Worth, TX: 1995.

2. Merrifield RB. Solid Phase Synthesis (Nobel Lecture). Angew. Chem. Int. Ed. 1985; 24:799-810.

3. Caruthers MH. Gene Synthesis Machines: DNA Chemistry and its Uses. Science. 1985; 230:281285. [PubMed: 3863253]

4. Seeberger PH, Haase W-C. Solid-Phase Oligosaccharide Synthesis and Combinatorial Carbohydrate Libraries. Chem. Rev. 2000; 100:4349-4393. [PubMed: 11749351]

5. Seeberger PH, Hicks MG, Kettner C. Bioinformatics - Key to the Future of Chemical Glycomics. Glyco-Bioinformatics: Bits 'n' Bytes of Sugars. 2009:25-36.

6. Plante OJ, Palmacci ER, Seeberger PH. Automated Solid-Phase Synthesis of Oligosaccharides. Science. 2001; 291:1523-1527. [PubMed: 11222853]

7. Evans DA, Bartroli J, Shih TL. Enantioselective Aldol Condensations. 2. Erythro-selective Chiral Aldol Condensations via Boron Enolates. J. Am. Chem. Soc. 1981; 103:2127-2129.

8. Paterson I, Scott JP. Laboratory Emulation of Polyketide Biosynthesis: An Iterative, Aldol-based, Synthetic Entry to Polyketide Libraries Using $(R)$ - and (S)-1-(benzyloxy)-2-methylpentan-3-one, and Conformational Aspects of Extended Polypropionates. J. Chem. Soc., Perkin Trans. 1999; $1: 1003-1014$.

9. Myers AG, Yang BH, Chen H, Kopecky DJ. Asymmetric Synthesis of 1,3-Dialkyl-Substituted Carbon Chains of any Stereochemical Configuration by an Iterable Process. Synlett. 1997; 36:457459.

10. Negishi E, Tan Z, Liang B, Novak T. An Efficient and General Route to Reduced Polypropionates via Zr-catalyzed Asymmetric C-C Bond Formation. Proc. Natl. Acad. Sci. U.S.A. 2004; 101:5782-5787. [PubMed: 15073327]

11. Thirsk C, Whiting A. Polyene Natural Products. J. Chem. Soc., Perkin Trans. 2002; 1:999-1023.

12. Rychnovsky SD. Oxo Polyene Macrolide Antibiotics. Chem. Rev. 1995; 95:2021-2040.

13. Cerullo G, Polli D, Lanzani G, De Silvestri S, Hashimoto H, Cogdell RJ. Photosynthetic Light Harvesting by Carotenoids: Detection of an Intermediate Excited State. Science. 2002; 298:23952398. [PubMed: 12493917]

14. Sklar LA, Hudson BS, Simoni RD. Conjugated Polyene Fatty Acids as Membrane Probes: Preliminary Characterization. Proc. Natl. Acad. Sci. U.S.A. 1975; 72:1649-1653. [PubMed: 1057769] 
15. Burton GW, Ingold KU. Beta-Carotene: An Unusual Type of Lipid Antioxidant. Science. 1984; 224:569-573. [PubMed: 6710156]

16. Luecke H, Schobert B, Richter H-T, Cartailler J-P, Lanyi JK. Structural Changes in Bacteriorhodopsin During Ion Transport at 2 Angstrom Resolution. Science. 1999; 286:255-260. [PubMed: 10514362]

17. Negishi, E-I. Handbook of Organopalladium Chemistry for Organic Synthesis. Vol. Vol. 1. John Wiley \& Sons; New York: 2002.

18. Nicolaou KC, Bulger PG, Sarlah D. Palladium-Catalyzed Cross-Coupling Reactions in Total Synthesis. Angew. Chem. Int. Ed. 2005; 44:4442-4489.

19. Miyaura N, Suzuki A. Palladium-Catalyzed Cross-Coupling Reactions of Organoboron Compounds. Chem. Rev. 1995; 95:2457-2483.

20. Mancilla T, Contreras R, Wrackmeyer B. New Bicyclic Organylboronic Esters Derived from Iminodiacetic Acids. J. Organomet. Chem. 1986; 307:1-6.

21. Gillis EP, Burke MD. A Simple and Modular Strategy for Small Molecule Synthesis: Iterative Suzuki-Miyaura Coupling of B-protected Haloboronic Acid Building Blocks. J. Am. Chem. Soc. 2007; 129:6716-6717. [PubMed: 17488084]

22. Gillis EP, Burke MD. Iterative Cross-Coupling with MIDA Boronates: Towards a General Strategy for Small Molecule Synthesis. Aldrichimica Acta. 2009; 42:17-27. [PubMed: 22523433]

23. Gillis EP, Burke MD. Multistep Synthesis of Complex Boronic Acids from Simple MIDA Boronates. J. Am. Chem. Soc. 2008; 130:14084-14085. [PubMed: 18837550]

24. www.aldrich.com/mida

25. Lee SJ, Gray KC, Paek JS, Burke MD. Simple, Efficient, and Modular Synthesis of Polyene Natural Products via Iterative Cross-Coupling. J. Am. Chem. Soc. 2008; 130:466-468. [PubMed: 18081295]

26. Woerly EM, Cherney AH, Davis EK, Burke MD. Stereoretentive Suzuki-Miyaura Coupling of Haloallenes Enables Fully Stereocontrolled Access to (-)-Peridinin. J. Am. Chem. Soc. 2010; 132:6941-6943. [PubMed: 20441218]

27. Fujii S, Chang SY, Burke MD. Total Synthesis of Synechoxanthin through Iterative CrossCoupling. Angew. Chem. Int. Ed. 2011; 50:7862-7864.

28. Brak K, Ellman JA. Total Synthesis of (-)-Aurantioclavine. Org. Lett. 2010; 12:2004-2007. [PubMed: 20356065]

29. Burns AR, McAllister GD, Shanahan SE, Tayler RJK. Total Synthesis and Structural Reassignment of (+)-Dictyosphaeric Acid A: A Tandem Intramolecular Michael Addition/Alkene Migration Approach. Angew. Chem. Int. Ed. 2010; 49:5574-5577.

30. Fujita K, Matsui R, Suzuki T, Kobayashi S. Concise Total Synthesis of (-)-Myxalamide A. Angew. Chem. Int. Ed. 2012; 51:7271-7274.

31. Li J, Burke MD. Pinene-Derived Iminodiacetic Acid (PIDA): A Powerful Ligand for Stereoselective Synthesis and Iterative Cross-Coupling of C(sp3) Boronate Building Blocks. J. Am. Chem. Soc. 2011; 133:13774-13777. [PubMed: 21823591]

32. Aridoss G, Zhou B, Hermanson DL, Bleeker NP, Xing CG. Structure-Activity Relationship (SAR) Study of Ethyl 2-Amino-6-(3,5-dimethoxyphenyl)-4-(2-ethoxy-2-oxoethyl)-4H-chromene-3carboxylate (CXL017) and the Potential of the Lead against Multidrug Resistance in Cancer Treatment. J. Med. Chem. 2012; 55:5566-5581. [PubMed: 22582991]

33. Gustafson JL, Lim D, Barrett KT, Miller SJ. Synthesis of Atropisomerically Defined, Highly Substituted Biaryl Scaffolds through Catalytic Enantioselective Bromination and Regioselective Cross-Coupling. Angew. Chem. Int. Ed. 2011; 50:5125-5129.

34. Grob JE, Nunez J, Dechantsreiter MA, Hamann LG. Regioselective Synthesis and Slow-Release Suzuiki-Miyaura Cross-Coupling of MIDA Boronate-Functionalized Isoxazoles and Triazoles. J. Org. Chem. 2011; 76:10241-10248. [PubMed: 22047083]

35. Coluccini C, Manfredi N, Salamone MM, Ruffo R, Grazia Lobello M, De Angelis F, Abbotto A. Quaterpyridine Ligands for Panchromatic Ru(II) Dye Sensitizers. J. Org. Chem. 2012; 77:79457956. [PubMed: 22917532] 
36. Kozhevnikov VN, Dahms K, Bryce MR. Nucleophilic Substitution of Fluorine Atoms in 2,6Difluoro-3-(pyridine-2-yl)benzonitrile Leading to Soluble Blue-Emitting Cyclometalated Ir(III) Complexes. J. Org. Chem. 2011; 76:5143-5148. [PubMed: 21612243]

37. Nishiyabu R, Kobayashi H, Kubo Y. Dansyl-containing Boronate Hydrogel Film as Fluorescent Chemosensor of Copper Ions in Water. RSC Adv. 2012; 2:6555-6561.

38. Dictionary of Natural Products. version 22.1. Taylor and Francis Group; 2013. dnp.chemnetbase.com.

39. Liu D, Li X-M, Meng L, Li CS, Gao S-S, Shang Z, Proksch P, Huang C-G, Wang B-G. Nigerapyrones A-H, a-pyrone Derivatives from the Marine Mangrove-Derived Endophytic Fungus Aspergillus niger MA-132. J. Nat. Prod. 2011; 74:1787-1791. [PubMed: 21774474]

40. Misono Y, Ito A, Matsumoto J, Sakamoto S, Yamaguchi K, Ishibashi M. Physarigins A-C, Three New Yellow Pigments from a Cultured Myxomycete Physarum rigidum. Tetrahedron Lett. 2003; 44:4479-4481.

41. Sakaki H, Kaneno H, Sumiya Y, Tsushima M, Miki W, Kishimoto N, Fujita T, Matsumoto S, Komemushi S, Sawabe A. A New Carotenoid Glycosyl Ester Isolated from a Marine Microorganism, Fusarium Strain T-1. J. Nat. Prod. 2002; 65:1683-1684. [PubMed: 12444702]

42. Hall, DG. Boronic Acids. Wiley-VHC; Weinheim, Germany: 2005.

43. Gray KC, Palacios DS, Dailey I, Endo MM, Uno BE, Wilcock BC, Burke MD. Amphotericin Primarily Kills Yeast By Simply Binding Ergosterol. Proc. Natl. Acad. Sci. U.S.A. 2012; 109:2234-2239. [PubMed: 22308411]

44. Kinzel T, Zhang Y, Buchwald SL. A New Palladium Precatalyst Allows for the Fast SuzukiMiyaura Coupling Reactions of Unstable Polyfluorophenyl and 2-Heteroaryl Boronic Acids. J. Am. Chem. Soc. 2010; 132:14073-14075. [PubMed: 20858009]

45. Knapp DM, Gillis EP, Burke MD. A General Solution for Unstable Boronic Acids: Slow-Release Cross-Coupling from Air-Stable MIDA Boronates. J. Am. Chem. Soc. 2009; 131:6961-6963.

46. Yuan W, Ma S. CuCl- $\mathrm{K}_{2} \mathrm{CO}_{3}$-Catalyzed Highly Selective Borylcupration of Internal Alkynes Ligand Effect. Org. Biomol. Chem. 2012; 10:7266-7268. [PubMed: 22872073]

47. Fang Z, Liao P-C, Yang Y-L, Yang F-L, Chen Y-L, Lam Y, Hua K-F, Wu S-H. Synthesis and Biological Evaluation of Polyenylpyrrole Derivatives as Anticancer Agents Acting Through Caspases-Dependent Apoptosis. J. Med. Chem. 2010; 53:7967-7978. [PubMed: 20964408]

48. Wang G, Huang Z, Negishi E-I. Efficient and Selective Syntheses of (all-E)- and (6E,10Z)-2'-OMethylmyxalamides D via Pd-Catalyzed Alkenylation-Carbonyl Olefination Synergy. Org. Lett. 2008; 10:3223-3226. [PubMed: 18593171]

49. Struble JR, Lee SJ, Burke MD. Ethynyl MIDA Boronate: A Readily Accessible and Highly Versatile Building Block for Small Molecule Synthesis. Tetrahedron. 2010; 66:4710-4718.

50. Imao D, Glasspoole BW, Laberge VS, Crudden CM. J. Am. Chem. Soc. 2009; 131:5024-5025. [PubMed: 19301820] 


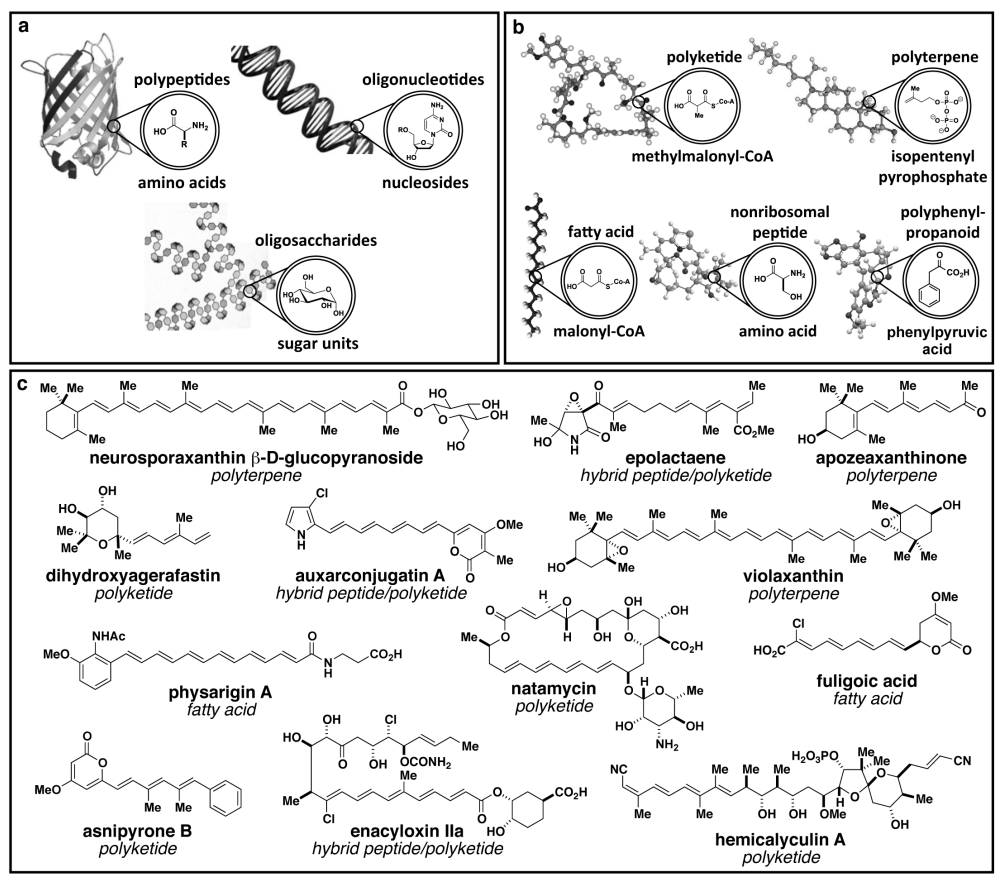

Figure 1.

The iterative assembly of bifunctional building blocks is a versatile strategy for the preparation of small molecules. a. Nature biosynthesizes macromolecules, including polypeptides, oligonucleotides, and oligosaccharides via the iterative coupling of building blocks. b. Nature similarly prepares small molecules derived from a range of biosynthetic pathways via the iterative assembly of bifunctional building blocks. c. A collection of polyene natural products from a variety of biosynthetic pathways. 

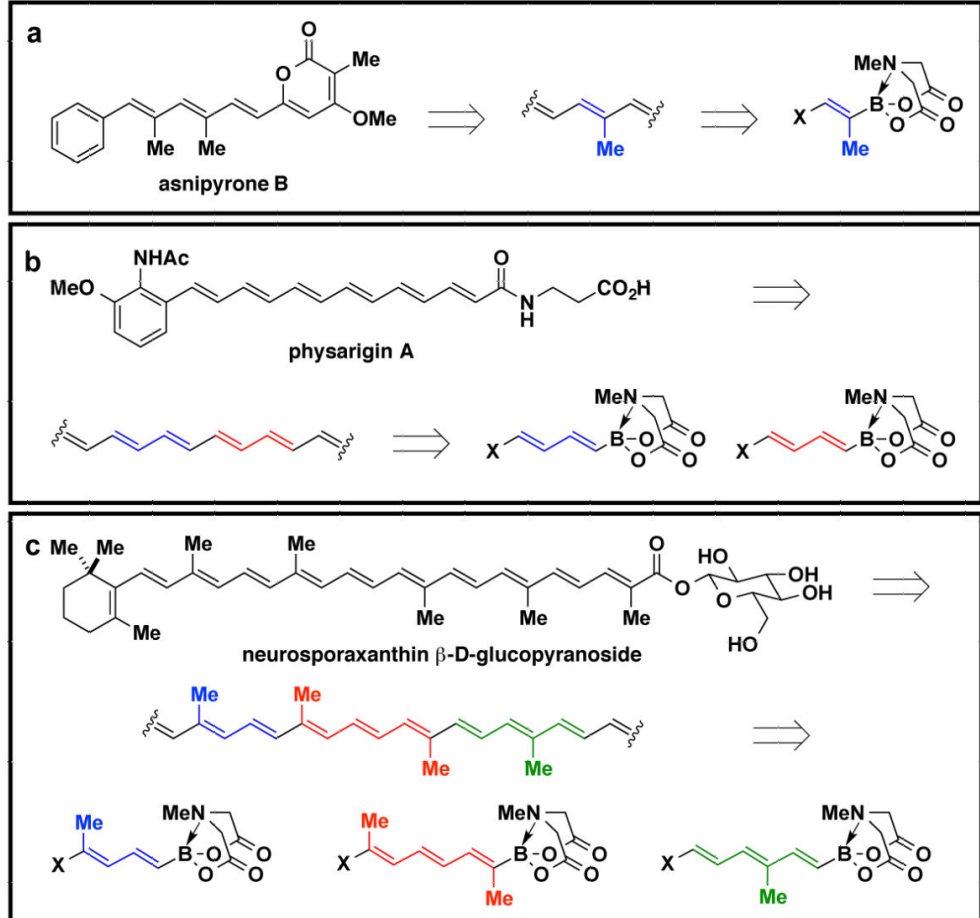

Figure 2.

Three examples of applying the standardized 3-step retrosynthetic algorithm to previously not synthesized polyene natural products. a. asnipyrone B. b. physarigin A. c. neurosporaxanthin $\beta$-D-glucopyranoside. 


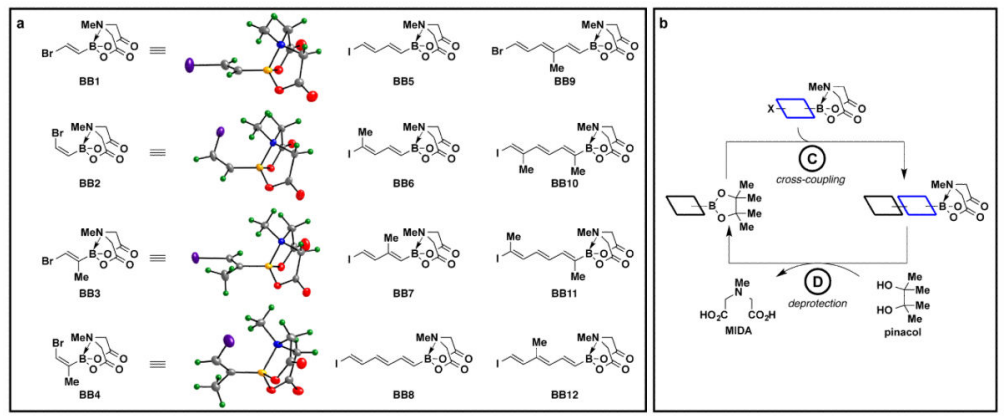

Figure 3.

A general platform for making polyene motifs via iterative cross-coupling. a. A collection of 12 bifunctional MIDA boronate building blocks BB1-BB12 can be used to synthesize the polyene motifs found in $>75 \%$ of all polyene natural products. b. An iterative cross-coupling strategy involving the coupling of a pinacol boronic ester to a bifunctional halo MIDA boronate building block followed by a deprotection of the resulting MIDA boronate to generate a new pinacol boronic ester suitable for the next round of coupling. 


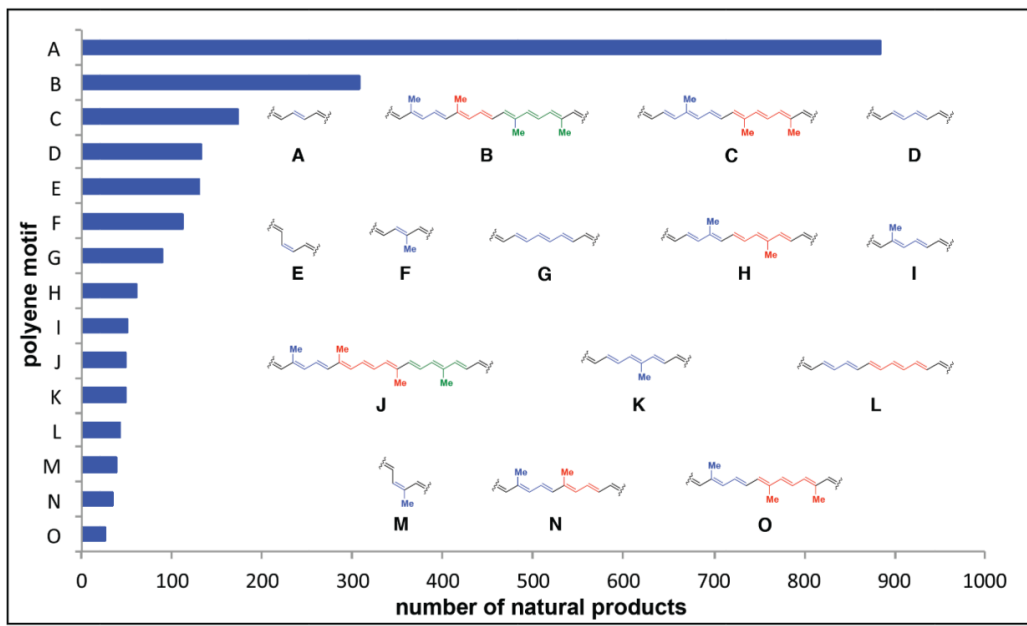

Figure 4.

A collection of polyene motifs collectively found in $>75 \%$ of all polyene natural products. 


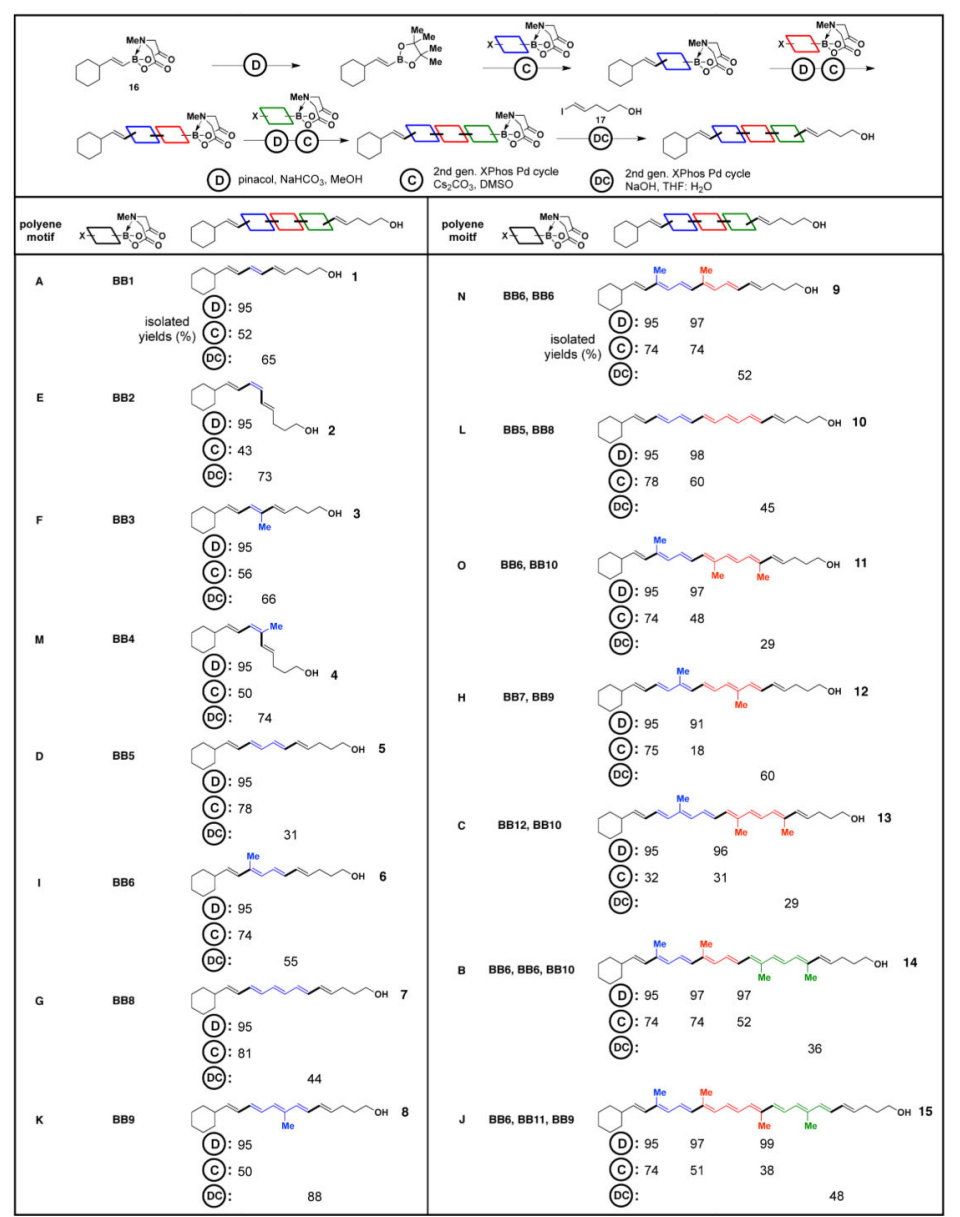

Figure 5.

The synthesis of polyene motifs present in $>75 \%$ of all known polyene containing natural products using just 12 bifunctional MIDA boronate building blocks and one coupling reaction. 


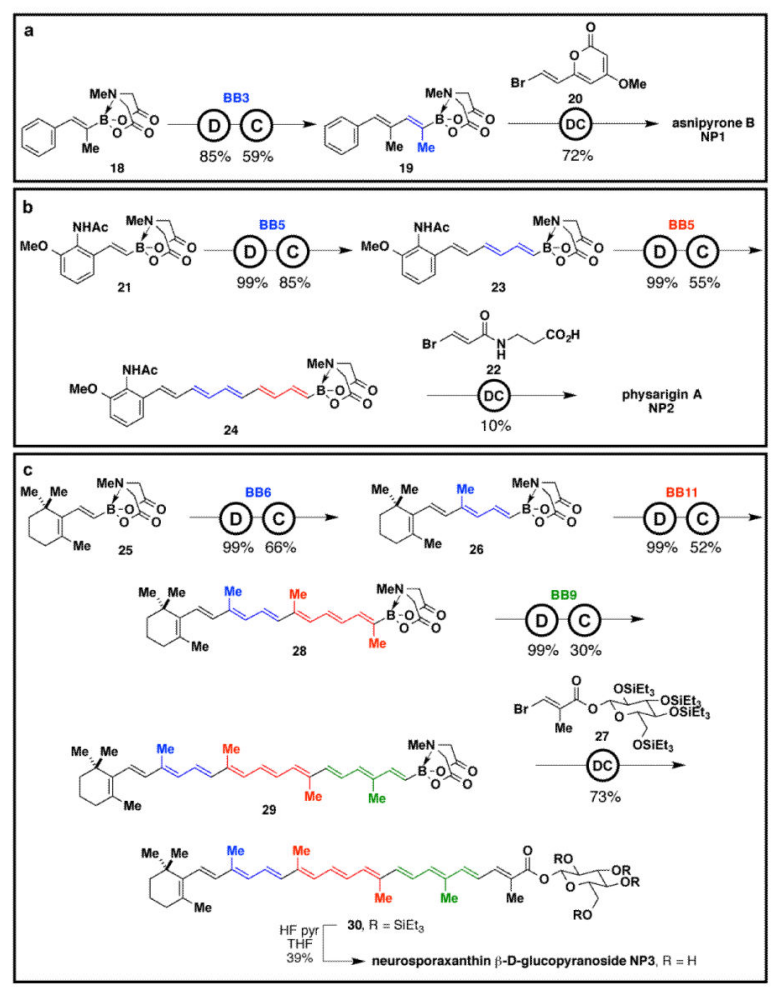

Figure 6.

The total synthesis of three polyene natural products using bifunctional MIDA boronate building blocks and one set of reaction conditions in an iterative fashion. a. The total synthesis of asnipyrone B. b. The total synthesis of physarigin A. c. The total synthesis of neurosporaxanthin $\beta$-D-glucopyranoside. 